\title{
Detection of malaria parasites in thick blood smear: A review
}

\author{
Faza Maula Azif*, Hanung Adi Nugroho, Sunu Wibirama \\ Department of Electrical Engineering and Information Technology, Universitas Gadjah Mada, Jl. Grafika No. 2, Yogyakarta 55281, Indonesia
}

Article history:

Received: 25 April 2018 / Received in revised form: 29 May 2018 / Accepted: 31 May 2018

\begin{abstract}
Based on data from World Health Organization, in 2015, there are $90 \%$ of deaths caused by malaria disease in Africa, Southeast Asia and countries of eastern Mediterranean. It makes the malaria become one of the most dangerous diseases that often leads to death. To support the diagnosis of malaria, early detection of plasmodium parasite is needed. Recently, malaria diagnosis process can be done with the help of computer, or often referred to as Computer Aided Diagnosis (CAD). By utilizing the digital image from the blood staining process, digital image processing can be performed to detect the presence of malaria parasite. There are 2 types of blood smear images that can be used in the malaria diagnosis process, namely, thin blood smear images and thick blood smear images. This paper provides a review of the techniques and methods used in the diagnosis of computer-assisted malaria using thick blood smear images as a diagnostic material.
\end{abstract}

Keywords: Malaria; microscopic image; red blood cells; object detection

\section{Introduction}

Transmission of malaria is caused by female anopheles mosquito bites. Mosquito habitats in tropical and subtropical areas make malaria as one of the health problems in the mentioned areas [1]. In 2015, World Malaria Report releases the data about the death cases that caused by malaria. It stated that 438.000 people in 200 million cases are dead because of malaria disease [2]. Most of the death cases that caused by malaria occur in Africa. This is because of the environmental conditions of some Africa regions that are favorable for mosquito's breeding. Moreover, social and economic conditions of African people who are still have difficulties to receive proper treatment and equipment in handling malaria resulting the high rate of death from malaria disease [2]. With the same region characteristics, eastern Indonesia also have the same problem, malaria is still one of the deathly infectious disease which become one of the health issue concern in Indonesia.

A gold standard for detecting and identifying the type and phase of malaria parasites in blood smear is visual identification by utilizing microscope. However, the interpretation of medical images is highly depend on human experience and expertise. Moreover, proper medical infrastructures are not available in all malaria endemic area [3]. To support medical personnel in making a decision of malaria diagnosis in mentioned area, an easy to use tool with fast and accurate diagnosis is needed [4].

Digital image processing becomes one of the most common computer-based methods which often used in

* Corresponding author.

Email: faza.maula.a@mail.ugm.ac.id computer aided diagnosis (CAD) [5]. By utilizing a computer with special software which developed to support the malaria diagnosis, it can be categorized as an easy tool that can be easily distributed in rural areas. Moreover, considering the variations of malaria cases in each region, by using a CAD technique with machine learning approach, the development of CAD software for malaria diagnosis can be improved time by time. Related research on this area has been widely done by several researchers. The aim of this article is to review some techniques and methodoligies of automated malaria diagnosis based on microscopic blood smear images.

\section{Malaria}

Malaria is caused by plasmodium parasite that is spread through the bites of female anopheles mosquito. When it spreads throughout the human body, plasmodium parasites will settle and multiply in the system of human liver while infecting red blood cells. There are 5 species of plasmodium that can cause malaria in human, they are plasmodium falciparum, plasmodium vivax, plasmodium ovale, and plasmodium malariae. In addition is the presence of malaria cases caused by plasmodium knowlesi in the forests of Southeast Asia, especially the island of Borneo.

\section{Detection of Plasmodium Malaria with Conventional Method}

The plasmodium parasite is a species lives in red blood cells. When infected human, it will multiply in red blood cells. To find out the existence of parasite in blood, experts will need equipment such as a microscope, to extract and enlarge blood images. These images are then analyzed in laboratory. 
Detection using microscope is an effective way of performing parasitic detection and still used until today.

\section{(1) Clinical Malaria Diagnosis.}

The clinical diagnosis of malaria is one of traditional malaria detection methods. The diagnosis is clinically done by observing the visible physical symptoms and characteristics of patient's body. Medics will try to identify the disease though early symptoms happened are still common, such as fever, headache, diarrhea, cold, chills, myalgia, abdominal pain, and vomiting [4].

The number of symptoms that experts need to consider in clinical diagnosis is leading to the difficulty for accurate identification of the disease, because the symptoms of the disease are likely still too general to determine whether that the patient has malaria or not.

\section{(2) Laboratory Malaria Diagnosis.}

There are several methods for diagnosing malaria in the laboratory. This kind of diagnosis requires special equipment, materials and expertise since mostly of these tools are only used by medical personnel. There are four commonly used methods for diagnosing malaria in the laboratory:

\section{a) Peripheral Blood Smear}

The diagnosis of malaria using PBS is based on blood smear. The presence of malaria parasites in the blood smear was observed using microscope. The blood smear can be thick and thin. The thick blood smear is used to detect the presence of plasmodium parasites, whereas the thin blood preparation is used to detect the type and phase of plasmodium [6].

The results of sensitivity and specificity depend on the technique used, the reagent, and the expertise of medical personnel. The required time to do the diagnosis is about $30-60$ minutes.

\section{b) Serological Tests}

Serologic examination is a test performed by recognizing human antibodies that work against parasites. The accuracy of the examination gives a quite good result [7]. Serologic examination is able to detect all types of plasmodium and very useful for epidemiological surveys. The disadvantage of this method is the required time to detect the parasite that is quite long, around 30-60 minutes.

\section{c) Quantitative Buffy Coat (QBC)}

Designed to improve malaria detection based on microscopic tests, QBC also makes easier malaria detection [7]. The sensitivity and specificity of QBC is better when compared to PBS and able to detect parasites less than 15 minutes. The QBC method is only able to detect specific types of plasmodium parasites.

\section{d) Rapid Diagnostic Tests (RDT)}

To solve the problems inflicted by microscopic tests, expertise designs a simple method that use antigens from parasites which identified through blood flow runs through membranes containing malarial antibodies.

This method does not require electricity or specialist expertise, and able to provide high accuracy detection results. However, RDT cannot determine the type of plasmodium parasite.

\section{(3) Staining Methods.}

Giemsa's stain has been applied for the first time for the diagnosis malaria since 1902. The giemsa blood staining is recognized by its low cost, high sensitivity and specificity, it is widely used in microscopical malaria examination [8]. However, giemsa staining requires multiple reagents, experienced personal and is labor-intensive and time consuming, typically, it requires at least 45 minutes to stain a slide [8].

To overcome the staining time, another staining method have been used, such as Field stain that significantly reduces the staining time, although it requires drying of samples before and during staining [9]. Even the staining time reduced, field's stain with poor blood preparation can generate artifacts. These artifacts commonly mistaken for malaria parasites.

A study by Sodeman et al. [10] investigated the effect of fluorochrome staining in identifying the malaria parasites at low-level infection. The result of the study is that fluorochrome staining is more sensitive and less timeconsuming than Romanowsky and Giemsa staining methods, but requires considerable practive and training, and suffers from artifacts including photobleaching and phototoxicity [11]. Another problem is fluorescence microscopes are more expensive than standard light microscopes, which is an important factor in tropical region with limited medical infrastructure [12].

Giemsa staining techniques and the use of thin blood smear dominated the publication in computer-aided malaria diagnosis. The easily visual identification of red blood cell in thin blood smear make the problem of parasite detection easier to a certain degree, as parasites need to be detected only inside cells.

Detection of malaria parasites in thick blood smear is more challenging, since the noise and artifacts that caused by the staining technique can lead to false positive detection. Because of the importance of thick blood smears for practical malaria diagnosis, a further research in this blood smear type is required.

\section{(4) Computer-based Malaria Diagnosis}

Diagnosis of malaria parasites can be done by performing visual identification of red blood cells. Malaria parasites will only infect red blood cells, especially the young red blood cells. This parasite will also infect the liver which is the place to produce red blood cells [13]

The use of digital image processing in Computer Aided Diagnosis (CAD) support the diagnosis procedure with the help from computer. Usually, the initial step of CAD is the acquisition of digital images of blood smears. This initial step breaks down the different approaches for the differenty types of microscopy, blood slides (thin or thick) and staining [14].

In general, the flow of digital image processing are: (1) image pre-processing, (2) image segmentation, (3) feature 
extraction, (4) classification based on similarity of object features.

\section{Methods}

\section{(1) Pre-processing}

In image processing, the initial stage that needs to be done is the pre-processing stage. Improving image quality and image size changes are done at this stage. The image that is being the object often has poor quality, for example the image has noises during the transmission through transmission line, the image brightness level, and the obscurity of the object in the image. Through this initial processing operation, the image quality is improved so that the image can be used for further applications.

Frean [15] used a rolling ball algorithm to clean and repair the background image. The parameter used in this algorithm was light background which produces a brighter background. Basically, this algorithm refines the image by reducing the noise in each area per $3 \times 3$ pixels. After the background became clearer, edge detection was done using sobel edge detector by highlighting the intensity changes that occur between objects and the background. The final stage of preprocessing in this research was to convert the image into black and white binary image and filled the holes that may appear on objects by using mathematical morphology.

Elter et al. [16] performed two major stages to detect malaria parasites. Pre-processing was in the first stage. According to this research, the proportion of red and green components of a blood smear image is the best feature for identifying objects which contain chromatin. Thus, in the preprocessing stage, this research tried to transform the original blood smear image into monochrome image, where the object containing chromatin turned into dark gray, and objects that did not contain chromatin became light gray.

Arco et al. [17] performed pre-processing which consist of 2 operations; image filtering to reduce noise, and improving image quality. At the noise reduction stage, a gaussian lowpass filter was used to get the signal region more clearly and suppress the effect of noise. Once the gaussian filter applied, the image became brighter, thus, the intensity of the pixels became higher than before. The next stage in the preprocessing was the histogram equalization. This operation produced images with high contrast. This method will be useful when the image is represented by adjacent contrast values. In this research, the adaptive histogram equalization method was used to calculate multiple histograms, in which each histogram was corresponding to different image sections, and used it to redistribute the brightness value of an image. The optimal value of the size of each image sub-division has been determined, 64 pixels $(8 \times 8)$ and bilinear interpolation of the pixel edge from 2 subdivisions has been calculated. Then, to prevent over-amplification of noise, contrast limits were used. After going through the image improvement process, an h-minima transformation operation was performed. This operation effectively suppressed all the intensity of minima in the image. With this operation, the difference between background pixels could be reduced.

Abidin et al. [18] did 2 processes of image quality improvement on image of ROI cutting result; noise filtering and contrast increasing. Noise filtering involved median filtering, Gaussian filtering, and lowpass filtering. Then, contrast enhancement was involving contrast stretching and dark stretching. Each method in both processes was combined into 6 combinations, including: median filtering - contrast stretching, gaussian filtering - contrast stretching, lowpass filtering - contrast stretching, median filtering - dark stretching, Gaussian filtering - dark stretching, and lowpass filtering - dark stretching.

Dave [19] converted RGB image to HSV. It was based on the assumption that the particles in the color space of HSV had significant differences and the image in the HSV color space could decrease brightness variation and blood staining concentration, which was very influential on the image with RGB color space. The channel used in the HSV color space is saturation channel. Median filter with a size of $3 \times 3$ then performed on the channel to reduce noise.

Because of the color variation as a result from differences in $\mathrm{pH}$ staining, age and purity of stain, duration of the staining procedure, and sensor settings, Mehanian et al. [20] use white balancing techniques to compensate some color variations. Basically, this white balancing technique is scaling red, green and blue (RGB) pixel values based on the mean color of the brightest pixels in each image individually [20]. The result of this technique is pixels from all fields of view pooled and global color balance affine transform for each blood sample is computed.

Rosado et al. [21] present different methodology approach for image processing of malaria-infected thick blood smears that are acquired with low cost and accessible tools such as smartphone. To replace microscope, this study use optical magnification prototype that can be easily adapted to a smartphone. The image processing software then installed in a smartphone application. A whole slide of thick blood smear images that acquired using this methodology has circular shape field of view with black background in each corner. In order to remove the circular shape, an optical circle detection is used in the firstplace. The second step of this study is to detect the white blood cell (WBC). Mean shift filtering was applied, this method can preserve edges and facilitate the segmentation as it preserves the edges of the WBC while the color inside the WBC nuclei becomes more homogeneous [21]. After all WBC detected, the last step is trophozoites detection, which is done by comparing the dimensions and stain contrast of trophozoites cytoplasm with WBC objects.

Salamah et al. [22] proposed two main steps image preprocessing. First step is contrast correction. Contrast correction is done globally and locally. For a global contrast correction, a dark stretching is performed, and for a local contrast correction, a contrast limited adaptive histogram equalization is used. After contrast of the image is corrected, the next step is to perform an edge correction, which conducted using unsharp masking filtering. Edge correction step is done by normalize the value of $R, G, B$ fom $[0,255]$ to $[0,1]$ and then convert the RGB image to HIS image. The next step is extracting the edge of the image using only the $I$ channel of the HIS image. The extraction process is done by blurring the image of the channel $I$ using gaussian blurring filter [22]. The main objective of the second step is to preserve the shape of the objects, so it can improve the visual 
impact on human vision and clarify contained information on the image.

Hanif et al. [23] performed dark stretching technique to enhance and to segment the parasite objects from thick blood smear image slide. To enhance the brightness and contrast level of the image, the auto scaling method which is a linear mapping function is used in dark stretching process [24]. This technique will be based on the original brightness and contrast level of the image to do the adjustment [24].

To enhance the contrast of the image, Kaewkamnerd et al. [25] controlled vertical movement of the motorized unit so that the system is able to capture images in different depths of field. The quality of the image is improved because of the margin in-focus information over a range of images to generate a single entirely in-focus image. To detect edges for in-focus pixel positions of each depth of field, laplacian spatial filter [26] is used because of its high accuracy and high speed.

Table 1. Summary of pre-processing techniques

\begin{tabular}{|c|c|c|}
\hline Challenges & $\begin{array}{c}\text { Pre-processing } \\
\text { methods }\end{array}$ & Remarks \\
\hline \multirow[t]{4}{*}{$\begin{array}{l}\text { Noise } \\
\text { reduction }\end{array}$} & $\begin{array}{l}\text { Rolling ball } \\
\text { algorithm [15]. }\end{array}$ & $\begin{array}{l}\text { Remove noises } \\
\text { while preserve } \\
\text { the edges. }\end{array}$ \\
\hline & $\begin{array}{l}\text { Gaussian low-pass } \\
\text { filter }[17,18] .\end{array}$ & \\
\hline & $\begin{array}{l}\text { Adaptive } \\
\text { histogram } \\
\text { equalization [17]. }\end{array}$ & $\begin{array}{l}\text { Very sensitive } \\
\text { to noises. } \\
\text { Requires image } \\
\text { smoothing } \\
\text { techniques so } \\
\text { that noises are } \\
\text { reduced. }\end{array}$ \\
\hline & $\begin{array}{l}\text { Median filtering } \\
{[18,19] .} \\
\text { Mean shift } \\
\text { filtering [21] } \\
\text { Laplacian spatial } \\
\text { filter [26]. }\end{array}$ & \\
\hline $\begin{array}{l}\text { Contrast } \\
\text { enhancement }\end{array}$ & $\begin{array}{l}\text { Dark stretching } \\
{[18,22] .}\end{array}$ & $\begin{array}{l}\text { Create brighter } \\
\text { image, objects } \\
\text { easily } \\
\text { recognized } \\
\text { visually. }\end{array}$ \\
\hline $\begin{array}{l}\text { Color } \\
\text { variation }\end{array}$ & $\begin{array}{l}\text { White balancing } \\
\text { [20] }\end{array}$ & $\begin{array}{l}\text { Compensate } \\
\text { color variation. }\end{array}$ \\
\hline
\end{tabular}

(2) Segmentation

Segmentation is the process of dividing the image into sections based on certain criteria. The main focus at this stage is the separation between the objects (white blood cells and plasmodium) and the background. Proper segmentation techniques will greatly impact the next stage and affect diagnosis accuracy.
The separation of the object against the background was done by Frean through particle analysis [15]. Initially, the particles considered as parasitic objects are selected manually by using point pickers. Point picker is a feature of the ImageJ software used in research by Frean [15].

Not only parasites which contain chromatin, but also leukocytes, platelets, and artifacts. Because of that, Elter et al. [16] separated plasmodium bacteria with leukocytes based on the characteristic of its former shape, while for platelets was using shape characteristics and intensity of staining. Blacktop-hat morphological operators and threshold count operation was used to separate plasmodium bacteria with other objects. Basically, morphological operator uses a general form that represents the target object to separate it with other objects. This research used the object with paraboloid shape with radius of 7 pixels, which was obtained from the size of plasmodium objects in general. The threshold value was specified with a fixed value, and performed globally. To combine the blobs contained in the binary image resulting from the threshold operation, the method of dilation with the circular form of element structure was used. The radius of this circular element structure was chosen to match the radius of the elemental structure used in black-top-hat operator.

The adaptive technique was again performed by Arco et al. [17] at the segmentation stage. In the previous process, this research produced an image histogram with unimodal characteristics, with several small peaks formed a large peak and unclear valleys. This made difficulties to determine the threshold value. To solve this problem, an adaptive thresholding process was used. First, image median was calculated with mean filter (15x15 mask). The median was then compared with the value of each pixel in the input image. Thus, there are two possibilities:

1) If the pixel in the original image is greater than $\mathrm{T} \%$ of the pixels in the image median, then the pixel is labeled as 1 , where label 1 is the background.

2) If the pixel in the original image is smaller than $\mathrm{T} \%$ of the pixels in the image median, then the pixel is labeled as 0 , where label 0 is a signal.

Before performing feature extraction, Arco et al. [17] performed mathematical morphological operations to improve the shape of objects in blood smear. There are 2 operations performed; dilation and erosion. Dilation operation was performed to close the hole, while erosion was to remove the irrelevant details of object. Then, object analysis based on interrelated components was done. This process aimed to select the objects of plasmodium candidates.

Abidin et al. [18] used image resulted from the selection of malaria parasite candidate in form of image with improved quality at pre-processing stage, to perform segmentation afterwards where active contour without edge (ACWE) is one of the main method of segmentation. The process of segmentation itself consists of 6 stages, they are:

\section{1) Complement}

It is the process of turning the image into negative image form. The image used in this research consists of dark-colored objects or parasites, and a background with brighter colors. Once the complement process is done, the object or parasite will turn brighter, and the background becomes darker. 


\section{2) $\mathrm{ACWE}$}

This algorithm is designed to segment objects that have obscure edge. In this research, it produced binary images with white objects and black backgrounds.

\section{3) Erosion + Dilation}

This stage aims to remove irrelevant small objects and refine the edges of object.

4) Masking

\section{5) Contrast stretching}

This stage aims to make the background darker and the object becomes lighter, resulting in more contrasting images.

\section{6) Thresholding}

By using a pre-determined thresholding value, this method is segmenting image into 2 areas, objects and background.

Dave [19] used adaptive histogram thresholding technique to separate parasitic candidate objects. The threshold value was derived from the search of cumulative distribution function value. At this stage, not all detected objects were malaria parasites. Thus, in the later stages, closing morphology operations was performed with structuring elements in disk shape to reduce false positive detection.

Several techniques are implemented by Mehanian et al. [20]. Before performing a segmentation step, Mehanian et al. [20], applied a dark threshold to a grayscale intensity image as an initial detector for malaria parasites candidates. The initial detector resulting in high sensitivity while its precision is low, large amount of distractor are also detected. These distractor objects cause the high number of false positive detections, which degrades the system performance. To overcome that problem, adaptive grayscale intensity and dynamic local thresholding are performed. The segmentation step which performed by Mehanian et al. [20] consists of two passes, first passes, white blood cell(WBC) candidates are segmented using dark threshold tied to grayscale intensity statistics. Individual WBC candidates are then filtered using morphological and clustering operations in order to classify each individual WBC using Gaussian-kernel SVM. The result of WBC segmentation is the RGB color collection statistics and a random sampling of background pixels. Another machine learning technique is implemented to compute the optimal projection in RGB space in order to separate WBC pixels from background pixels.

Segmenting the WBC objects is an important part of the study by Rosado et al. [21]. The segmentation technique which used in this study is adaptive thresholding from the mean intensity value of the square region centered on the pixel location. Once the WBC objects detected, the malaria parasite candidates can be detected by comparing the dimensions and stain contrast of trophozoite cytoplasm, then area filtering process was applied to get the trophozoite objects.

After pre-processing step is done, Kaewkamnerd et al. [25] convert the whole in-focus image into HSV (Hue-SaturationValue) color format. For segmentation purposes, the value component from HSV color channel is employed. The segmentation is done in three steps, first, construct a histogram of value components and extract non-background objects using adaptive threshold [27, 28] based on the histogram value. Second step is to divide the image into small windows of 300 by 300 pixels for efficient use of resources in searching process. The connected component analysis then performed in order to labelling each object for future reference. The last step is filtering malaria parasites from other objects according to their difference in sizes. The second and third steps are repeated until all the parasites are discovered and labelled [25].

Table 2. Summary of segmentation techniques

\begin{tabular}{|c|c|}
\hline $\begin{array}{l}\text { Segmentation } \\
\text { techniques }\end{array}$ & Remarks \\
\hline $\begin{array}{l}\text { Black-top-hat } \\
\text { morphology [16] }\end{array}$ & $\begin{array}{l}\text { With correct parameters, this } \\
\text { morphological operators can } \\
\text { remove WBC objects. } \\
\text { However, in certain case, it } \\
\text { can increase the false } \\
\text { positive number. }\end{array}$ \\
\hline $\begin{array}{l}\text { Global Threshold } \\
{[18]}\end{array}$ & $\begin{array}{l}\text { Threshold value applied to } \\
\text { all slide image without } \\
\text { considering the image } \\
\text { characteristics. }\end{array}$ \\
\hline $\begin{array}{l}\text { Adaptive } \\
\text { thresholding }[17 \text {, } \\
19,21,25]\end{array}$ & $\begin{array}{l}\text { The threshold value is } \\
\text { determined based on image } \\
\text { calculation, such as } \\
\text { histogram, mean intensity } \\
\text { value. }\end{array}$ \\
\hline $\begin{array}{l}\text { Dilation + Erosion } \\
{[17]}\end{array}$ & $\begin{array}{l}\text { Holes filling and remove } \\
\text { small objects. }\end{array}$ \\
\hline $\begin{array}{l}\text { Active Contour } \\
{[18]}\end{array}$ & $\begin{array}{l}\text { Very effective with uniform } \\
\text { lightning condition, the curve } \\
\text { evolution enables whole } \\
\text { object capture with similar } \\
\text { shape. } \\
\text { Heavy calculation. }\end{array}$ \\
\hline
\end{tabular}

(3) Feature extraction

After objects are separated from the background, feature extraction is applied to those objects to recognize objects based on feature similarities.

In a research conducted by Frean [15], after the object coordinates were obtained, the analysis of the object was done by using particle analysis to obtain object characteristics, so the calculation can be done automatically. This particle analysis feature requires object's size parameter and level of circle. Particle analysis generates the size of object that most likely is the characteristic of malaria parasite object.

Elter et al. [16] performed feature extraction in the first (detection) and second (error detection reduction) stage. The feature extraction in the first stage was performed to extract the positions of plasmodium candidates using a simple connected component labeling algorithm. The extracted objects were determined as the positions of plasmodium candidates based on their centroid. In the second stage, feature extraction was performed by cutting the region of interest (ROI) in $80 \times 80$ pixels on each plasmodium candidate from the 
input image. Based on these ROI image pieces; statistical features, texture analysis features, and color textures were used as a set of features to be extracted. It generated a large set of features. These features were then selected to produce an optimized feature subset using univariate rankings to select the top 60 subsets that have great correlation with objects and genetic algorithms, to minimize features generated by univariate ratings.

After the objects of the candidate of plasmodium are filtered, Arco et al. [17] performed object property measurements. The main difference between white blood cells and malaria parasites is their size, thus, the size became one of the properties used. Then, the entire area (the number of pixels forming the area) was checked, resulting in 2 parts; parasites or white blood cells, which in this way, the higher the value of the label given to a field indicated the number of fields (parasites and white blood cells) in the image.

The cutting of objects detected as parasitic candidates is done by Dave [19], to perform feature extraction based on the result of the cutting of the object afterwards. The feature extraction of each object produced 123 features that each object had. Features possessed by each object were 56 statistical features, 16 geometry textures, 24 intensity textures, 22 color features, and 1 frequency domain feature.

Based on Mehanian et al. [20] paperwork, objects that survived the distractor filter are then assumed as malaria parasite candidates. Because of the proposed method is mainly about Convolutional Neural Network (CNN), it performed some experiments using deep learning framework, including Alexnet, VGG and GoogleNet. After subsequent experiments, it comes to a conclusion that VGG feature extractor is better than Alexnet and GoogleNet. To avoid overfitting when training the $\mathrm{CNN}$, this paperwork perform an augmentation of data by randomize gamma correction of individual color channels. This process gave more realistic blood smear microscopy image colors as well as improved performance [20].

Rosado et al. [21] Extract WBC and trophozoites candidates. For each WBC candidate, a total of 152 image features were extracted and grouped into 3 major groups: Geometry, Color, and Texture features. For each trophozoites candidates, a total of 314 features are extracted. 10 ratios between specific cytoplasm and chromatine features such as area, maximum and minimu diameter, perimeter, convex hull area, bounding box area are also extracted.

Purnama et al. [29] perform a manual crop of parasite objects as a region of interest. Extracted features from cropped images are mean, standard deviation, kurtosis, skewness and entropy of the red-green-blue channel color histogram, hue channel of the HSV histogram, and hue channel of HSI histogram.

\section{(4) Classification}

The similarity of features between objects obtained in the previous process then classified to recognize objects based on feature similarity, so that whether the object is a malaria parasite or other artifact in the blood can be determined.

Frean [15] again performed particle analysis with parameters that already obtained in the previous feature extraction process. After the median size of malaria parasite was determined, this study again conducted a particle analysis to separate malaria parasite with other artifacts.

Table 3. Summary of extracted feature

\begin{tabular}{|c|c|}
\hline Author & Extracted feature \\
\hline Frean [15] & $\begin{array}{l}\text { Object size and circular } \\
\text { level. }\end{array}$ \\
\hline Elter et al. [16] & $\begin{array}{l}\text { Statistical features, texture } \\
\text { analysis features, and color } \\
\text { textures }\end{array}$ \\
\hline Arco et al. [17] & Object size \\
\hline Dave [19] & $\begin{array}{l}\text { Statistical, Geometry, } \\
\text { Intensity, Color, Frequency } \\
\text { domain. }\end{array}$ \\
\hline $\begin{array}{l}\text { Mehanian et al. } \\
{[20]}\end{array}$ & $\begin{array}{l}\text { Using CNN framework such } \\
\text { as Alexnet, GoogleNet, } \\
\text { VGG. }\end{array}$ \\
\hline Rosado et al. [21] & $\begin{array}{l}\text { Geometry, Color, and } \\
\text { Texture features. }\end{array}$ \\
\hline $\begin{array}{l}\text { Purnama et al. } \\
\text { [29] }\end{array}$ & $\begin{array}{l}\text { Mean, standard deviation, } \\
\text { kurtosis, skewness and } \\
\text { entropy of color channel } \\
\text { combination. }\end{array}$ \\
\hline
\end{tabular}

Elter et al. [16] used Support Vector Machine (SVM) algorithm to perform the classification. Once a feature subset was found, SVM algorithm was used to study it, then classified it by 2 classes; plasmodium or artifacts.

Arco et al. [17] used the opening operation to remove white blood cells, resulting in images containing only malaria parasites. The structural size of the element of average white blood cell size had been determined, so that all elements smaller than the specified value will be removed. This step produced an image that contains only white blood cells. By calculating the difference between the two images, the original image with the image containing the white blood cells produced an image containing only malaria parasites.

Dave [19] classified it into 5 classes; ring form, early trophozoite (ET) form, late trophozoite (LT) form, leucocytes, and non-parasites (artifacts, platelets and other particles). The LT class consists of two parasitic phases; schizont and gametocyte, because these two parasitic phases are difficult to be recognized. Support vector machine (SVM) was used to classify, extracted 123 features from 2323 ROIs (tested results from 43 image data trained) and tested in 2290 ROIs (result of cutting object from 44 image data test). In this research, the more features used will improve accuracy. Parasitic calculations by proposed algorithms were able to detect parasites in ring, trophozoite, schizont and gametocyte phases. Proposed algorithm error rate was $7.14 \%$ and still in WHO quality control limit.

Mehanian et al. [20] implement a logistic regression as the external classifier. The reasons behind the use of logistic regression as external classifier are mimics the CNN fullyconnected + SoftMax output and the software package implements a robust, large-scale learning algorithm for logistic regression based on stochastic gradient descent [20]. 
Rosado et al. [21] performed 2 classifications. First, to classify WBC and second, trophozoites classification. WBC and trophozoites classification used the same classification method. A two-class SVM classifier with a RBF kernel was used to create a classification model, using a grid-search approach to obtain the best parameters [21].

Kaewkamnerd et al. [25] classify the malaria parasites into two species, $P$. falciparum $(\mathrm{Pf})$ and $P$. vivax $(\mathrm{Pv})$ based on chromatin size feature. The observation of the chromatin size shows that Pf parasites have smaller size of chromatin than those of Pv. To verify this argument, chromatin size of a total 4.000 samples of both parasite species were investigated. The decision process was then performed by evaluating the distribution of chromatin size as in the following criteria [25]:

- Number of parasite =0: classified as no infection

- Chromatin size < $64.5 \mathrm{~nm}$ : classified as unknown object

- $64.5 \mathrm{~nm}<$ chromatin size < $258 \mathrm{~nm}$ : classified as Pf parasite

- $\quad 64.5 \mathrm{~nm}<$ chromatin size $<688 \mathrm{~nm}$, and the amount of chromatin size of $>258 \mathrm{~nm}$ greater than 20 percents: classified as $\mathrm{Pv}$ parasite.

Purnama et al. [29] proposed a genetic programming (GP) method to classify parasites and non parasites objects. GP is a method to evolve computer programs [30]. Automatic programming requires developing a computer program that can produce a desired output for a given set of inputs [31]. To represent the evolutionary computation, GP used tree and graph. This method is usually used for machine learning.

Table 4. Summary of classification methods

\begin{tabular}{ll}
\hline \multicolumn{1}{c}{ Author } & \multicolumn{1}{c}{ Classification method } \\
\hline Frean [15] & Particle analysis. \\
Elter et al. [16] & SVM \\
Arco et al. [17] & Connected component \\
& labelling filter. \\
Dave [19] & SVM. \\
Mehanian et al. [20] & CNN. \\
Rosado et al. [21] & SVM. \\
Purnama et al. [29] & Genetic programming. \\
\hline
\end{tabular}

\section{Discussion}

In the early stages of Frean's experiment [15], to obtain a particle analysis algorithm that can be used in thin blood smear, user intervention was needed in adjusting the various algorithmic variables when analyzing blood preparations with low parasitic density. Despite the high accuracy and reliable results, Frean's research had not been fully automated. In this research, Frean [15] also stated that the staining process of high quality blood contributed to result in high accuracy. With the conditions in malaria endemic areas where limited medical infrastructure, image of good quality blood smear is still difficult to be obtained.

In blood smear with low plasmodium density, the approach used by Elter et al. 16] was capable of providing high detection sensitivity (97\%) with low false positive detection in each image $(8 \%)$. This research used the image of blood preparation in above standard quality, with little amount of parasite density in the blood smear (less than 5). Separation of plasmodium with artifacts was also not performed, and could lead to failure in detecting parasites and artifacts. The used of black-top-hat morphological surgery was able to distinguish between malaria parasites and background well. This research used an image with a low parasitic density (3 parasites in 1 image for average). A high degree of sensitivity which lead to failure in malaria parasite detection was completed by the second stage; reduced sensitivity by classifying detected region of interest based on statistical features, textures and colors. The feature extraction based on 3 object characteristics highly depends on the segmentation stage (separation of object and background). If in the segmentation stage does not produce object's separation in good result (for example, the actual form of a parasite does not resemble its original form), feature extraction will not produce good features for classification, thus impacting classification accuracy.

According to Arco et al. [17], to calculate the number of parasites accurately and rapidly in digital images, this research can use adaptive histogram equalization and adaptive thresholding approach. Certain traits of the image will lead to misinterpretation of parasites and increase the calculation mismatch between manual and digital counting. The application of the proposed method yields an average accuracy rate of up to $96.46 \%$ with low processing time (2 seconds of each image on a medium computing platform). The malaria parasite objects in the image used in this study have the same shape when converted into binary (white dots), therefore the connected component labeling algorithm provides good results for object classification. Implementation of connected component labeling algorithm can give unfavorable results if the form of the object varies. Variations in the shape of an object usually form at a low parasite density.

The research conducted by Abidin [18] tried to use the ACWE segmentation approach that matched the characteristics of to-be-processed low quality image with unclear parasite form. In this research, the combination of image quality improvement between lowpass filtering and contrast stretching provides the best quality image results. The accuracy of segmentation in this research reached $97.57 \%$ with false-negative rate is $12.04 \%$. This study cuts the ROI object from a thick blood smear image, for pre-processing and segmentation afterwards. The implementation of ACWE segmentation is not performed on the whole field of thick blood smear images. This raises the question of whether this segmentation technique would be effective when used in the whole field of blood smear image, taking the characteristics of the active contour method into account, which requires masking initiation at first, author's assumption is that this masking initiation will impact the 2 segmentation stages; the first stage is the segmentation to get ROI, and the second stage is to improve the shape of previously segmented objects. To think that computation time of ACWE method is quite high, the addition of segmentation in the early stages will provide additional computing time.

The classification of parasite into 5 classes performed by Dave [19] did not publish the points of accuracy. However, this study opens the possibility of identifying the phase of malaria parasite in the thick blood smear that is usually performed on a thin blood smear. 
The promising results of computer aided diagnosis for malaria in thick blood smear images is done by Mehanian et al. [20]. The proposed method is working well because the use of large amount of thick blood smear image data which suitable for neural-network based method. In this case, CNN is used to classify between parasites and non-parasite objects. Evaluation of the proposed method is done in two ways, patient-level and object-level. The results indicate that the system has achieved malaria diagnosis accuracy sufficient to attain competence level 1 in the WHO external competency assessment of malaria microscopists for P. falciparum, which means, that it perform a par with well-trained microscopists for this species [20]. However, the system is working well with high density of parasite. Malaria parasite quantitation is sufficiently accurate for parasitemia $>1000 \mathrm{p} / \mu \mathrm{L}$ and supported by large amount of data. Exploration of the proposed method in low parasite density and the use of limited data is still open for a further research. Moreover, the high quality of the images also has a big impact on the proposed method to give good results.

A mobile-based framework for malaria parasites detection has developed by Rosado et al. [21]. The classification results are presented in terms of three metrics: sensitivity, specificity and accuracy. Automatic detection of WBC in thick blood smears achieved $98.2 \%$ of sensitivity and $72.1 \%$ specificity, while the P.falciparum trophozoites detection achieved a sensitivity of $80.5 \%$ and a specificity of $93.8 \%$. In this study, the focus of the detection are WBC and trophozoites, which can be easily segregated based on its characteristics. Moreover, the blood staining process can emerge artefact object that has similar characteristics with trophozoites which is not considered to be an object to be detected. This issue can lead to false detection of trophozoites and reduce the specificity.

The adaptive local contrast correction to enhance image quality by Salamah et al. [22] is very sensitive to noise. In other words, the proposed scheme is dependent on the image smoothing technique. The smoothing technique should be as good as possible to remove noises so that the adaptive local contrast correction can performed optimally. The evaluation of the enhanced image is done using Minimum Square Error (MSE) and Peak Signal to Noise Ratio (PSNR). The value of MSE and PSNR is better if the range of the image histogram is short [22]. Based on this finding, Salamah et al. [22] stated that the proposed method is effective if the contrast of original image is low.

The result of dark stretching technique which proposed by Hanif et al. [23] is the stretched dark areas of the image while bright areas are compressed. In the thick blood smear images, dark area is referred to the parasites, thus the parasites become clearer due to the stretching step in dark stretching technique [23]. In order to find the suitable threshold value, threshold parameter value had been applied in different value, meanwhile the dark stretching factor only used the same parameter for all images, which is 255 . The evaluation of the dark stretched image is done by visual inspection. The darker objects and the brighter background show that the proposed image enhancement technique is well performed.

The proposed approach by Kaewkamnerd et al. [25] to detect and classify malaria parasites is done on 60 giemsa- stained thick blood films (40 blood films containing infected red blood cells and 20 control blood films of normal red blood cells). The proposed method detected parasite-positive and parasite-negative blood films at the rate of $95 \%$ and $68.5 \%$ accuracy, respectively. The performance of the proposed classification was correctly classified with the success rate of $75 \%$ while the accuracy of Pf classification was $90 \%$. Images that are acquired by motorized units for controlling the movements of objective lense and microscope stage can produce high quality image slide, while, sometimes it is quite difficult to have such motorized unit to produce high quality image. With the good quality of the image, the end result of parasite detection or classification will have a promising result.

Purnama et al. [29] classified all detected object into two and six classes. Classification process performed using GP method for 120 training data and 60 testing data. The result of the classification shows that the two-class classification has higher accuracy than six class classification. The combination between color channels which performed in this study is interesting idea and it needs to do more exploration.

\section{Conclusion}

Research to detect malaria parasites in blood smear has been widely practiced. Before determining what method to use, previous researches identified image characteristics which are going to be processed first.

Stages of sequential image processing have an impact on the importance of pre-processing as the initial stage that will affect the next stages. The adaptive technique proposed by Arco et al. [17] provides promising results, both in the preprocessing stage and in the segmentation stage. However, the characteristics of the image used will greatly affect the detection result. Moreover, in his research, Arco et al. [17] assumed that all objects with area value smaller than detected white blood cells are parasites, in fact, there is possibility that some objects that have similar area value as parasites are artefacts.

The use of images with different characteristics in each research has an impact on the difficulties to determine which method is best to use. This suggests that the proposed method in this field of research is highly depends on the characteristics of the image used. The exploration of method or scheme of optimal image processing sequence in a particular image characteristic can still be deeply explored for further research. Moreover, the majority of the publication in thick blood smear do not separate malaria parasite with artefacts that might be appear as a result of staining process.

The limited data of medical image in each study is one factor that restricts the researchers in conducting further exploration. When medical image data can be easily obtained, it opens the possibility to explore with deep learning algorithm. Several studies $[31,32,33]$ in the topic of malaria identification began to use deep learning, but the image used was still from thin blood smear.

\section{References}

1. WHO World Malaria Report 2015 Key points, World Health Organization, pp. X, 2015. 
2. Hanung Adi Nugroho, Son Aji Akbar, "Feature Extraction and Classification for Detection Malaria Parasits in Thin Blood Smear", Inf Technol. Comput. Electr. Eng., vol. 2, 2015, pp. 197-201.

3. S. A. Nasir, M. Y. Mashor, Z. Mohamed, "Segmentation based approach for detection of malaria parasits using moving k-means clustering", IEEE EMBS Conf. Biomed. Eng. Sci., 2012, pp. 653-658.

4. Saputra, W. A., Nugroho, H. A., \& Permanasari, A. E. Toward development of automated plasmodium detection for Malaria diagnosis in thin blood smear image: An overview. 2016 International Conference on Information Technology Systems and Innovation, ICITSI 2016 Proceedings.

5. J. Somasekar, B. Eswara Reddy, "Segmentation of erythrocytes infected with malaria parasits for the diagnosis using microscopy imaging", Computers \& Electrical Engineering vol 45, 2015, pp. 336-351.

6. She, R. C., Rawlins, M. L., Mohl, R., Ascp, M. T., Perkins, S. L., Hill, H. R., \& Litwin, C. M. (2007). "Comparison of Immunofl uorescence Antibody Testing and Two Enzyme Immunoassays in the Serologic Diagnosis of Malaria", 14(2), 105-111.

7. Thomas E. Clendennen III., Gary W. Long., J.Kevin Baird, QBC and Giemsa-stained thick blood films: diagnostic performance of laboratory technologists", Transactions of the Royal Society of Tropical Medicine and Hygiene, 1995, pp. 183-184.

8. Keiser J, Utzinger J, Premji Z, Yamagata Y, Singer BH. Acridine orange for malaria diagnosis: its diagnostic performance, its promotion and implementation in Tanzania, and the implications for malaria control. Ann Trop Med Parasitol 2002;96:643-54. Taylor \& Francis.

9. Houwen, B. Blood film preparation and staining procedures. Clin Lab Med 2002;22:1-14. Elsevier.

10. Shute G, Sodeman T. Identification of malaria parasites by fluorescence microscopy and acridine orange staining. BullWorld Health Organ 1973;48-50:591-6.

11. Suwalka, I., Sanadhya, A., Mathur, A., \& Chouhan, M. S. (2012). Identify malaria parasite using pattern recognition technique. 2012 International Conference on Computing, Communication and Applications, 1-4.

12. Diaspro A, Chirico G, Usai C, Ramoino P, Dobrucki J. Photobleaching. In: Handbook of biological confocal microscopy. Springer; 2006:690702 .

13. Iis Hamsir Ayub Wahab, Adhi Susanto, "Deteksi Parasit Malaria Dalam Sampel Darah Untuk Menunjang Keperluan Diagnosis Berbasis Data Ciri Tekstur Warna", Dissertation Thesis, Universitas Gadjah Mada, Indonesia, 2015.

14. Poostchi, M., Silamut, K., Maude, R. J., \& Jaeger, S. (2016). Image analysis and machine learning for detecting malaria. Translational Research, 194, 36-55.

15. Frean JA, Reliable enumeration of malaria parasites in thick blood films using digital image analysis. Malar J 8: 218 (2009).

16. Elter, M., Haßlmeyer, E., \& Zerfaß, T. Detection of malaria parasites in thick blood films. Proceedings of the Annual International Conference of the IEEE Engineering in Medicine and Biology Society, 2011, EMBS, 5140-5144.

17. Arco, J. E., Górriz, J. M., Ramírez, J., Álvarez, I., \& Puntonet, C. G. (2015). Expert Systems with Applications Digital image analysis for automatic enumeration of malaria parasits using morphological operations. Expert System With Application, 42(6), 3041-3047.

18. S. R. Abidin, U. Salamah and A. S. Nugroho, "Segmentation of malaria parasite candidates from thick blood smear microphotographs image using active contour without edge," 2016 1st International Conference on Biomedical Engineering (IBIOMED), Yogyakarta, 2016, pp. 1-6.

19. I. R. Dave, "Image analysis for malaria parasite detection from microscopic images of thick blood smear," 2017 International Conference on Wireless Communications, Signal Processing and Networking (WiSPNET), Chennai, 2017, pp. 1303-1307.

20. C. Mehanian et al., "Computer-Automated Malaria Diagnosis and Quantitation Using Convolutional Neural Networks," 2017 IEEE International Conference on Computer Vision Workshops (ICCVW), Venice, 2017, pp. 116-125.

21. Correia, M., Elias, D., \& Cardoso, J. S. (2016). Automated detection of malaria parasites on thick blood smears via mobile devices, 90(July), 138-144.

22. U. Salamah et al., "Enhancement of low quality thick blood smear microscopic images of malaria patients using contrast and edge corrections," 2016 International Conference on Knowledge Creation and Intelligent Computing (KCIC), Manado, 2016, pp. 219-225.

23. N. S. M. M. Hanif, M. Y. Mashor and Z. Mohamed, "Image enhancement and segmentation using dark stretching technique for Plasmodium Falciparum for thick blood smear," 2011 IEEE 7th International Colloquium on Signal Processing and its Applications, Penang, 2011, pp. 257-260.

24. N.A.Mat Isa, M.Y.Mashor \& N.H.Othman, "Contrast Enhancement Image Processing Technique on Segmented Pap Smear Cytology Images", Proc. of Int. Conf.on Robotics, Vision, Information and Signal Processing, 118-125, 2003.

25. Kaewkamnerd S, Uthaipibull C, Intarapanich A, Pannarut $M$, Chaotheing S, Tongsima S. An automatic device for detection and classification of malaria parasite species in thick blood film. BMC Bioinformatics 2012;13:S18.

26. Peli E. Contrast in complex images. J Opt Soc Am A. 1990;7:20322040. doi: 10.1364/JOSAA.7.002032.

27. Haralick R, Shapiro LG. Computer and Robot Vision.Addison_Wesley Publishing Company; 1992.

28. Shapiro LG, Stockman GC. Computer Vision. Prentice Hall; 2002.

29. I. K. E. Purnama, F. Z. Rahmanti and M. H. Purnomo, "Malaria parasite identification on thick blood film using genetic programming," 2013 3rd International Conference on Instrumentation, Communications, Information Technology and Biomedical Engineering (ICICI-BME), Bandung, 2013, pp. 194-198.

30. Koza, J. R. Genetic Programming: On the Programming of Computers by Means of Natural Selection. The MIT Press, 1992.

31. J.R. Koza, "Hierarhical Genetic Algorithms Operating on Populations of Computer Programs", Morgan Kaufmann Publishers Inc. San Francisco, CA, USA, 1989.

32. Z. Liang et al., "CNN-based image analysis for malaria diagnosis," 2016 IEEE International Conference on Bioinformatics and Biomedicine (BIBM), Shenzhen, 2016, pp. 493-496.

33. Y. Dong et al., "Evaluations of deep convolutional neural networks for automatic identification of malaria infected cells," 2017 IEEE EMBS International Conference on Biomedical \& Health Informatics (BHI), Orlando, FL, 2017, pp. 101-104.

34. Gopakumar GP, Swetha M, Sai Siva G, Sai Subrahmanyam Gorthi R. $\mathrm{K}$. Convolutional neural network-based malaria diagnosis from focus stack of blood smear images acquired using custom-built slide scanner. J. Biophotonics. 2018 